\title{
Las encuestas de movilidad y los referentes ambientales de los transportes
}

Carme Miralles-Guasch. Universidad Autónoma de Barcelona, Barcelona, España.

RESUMEN | La contribución de los desplazamientos de la población a las emisiones de gases de efecto invernadero, en la medida en que utiliza medios de transporte mecánicos, principalmente el automóvil, es irrefutable. Muchos trabajos coinciden en que esa contribución bordea el 30 por ciento del total de emisiones. Sin embargo, no todos los medios de transporte tienen un mismo nivel de incidencia en este proceso, por lo que es de suma importancia analizar el reparto modal de los movimientos de una población para determinar su grado de participación en la contaminación ambiental. Este artículo analiza el reparto modal en Cataluña, partiendo de los datos de la Encuesta de Movilidad Cotidiana (2006), desde el binomio territorial urbano/rural y desde la categoría motivacional que los genera.

PALABRAS CLAVE | Movilidad, transporte urbano, medio ambiente

ABSTRACT | The contribution of the population trips to the emission of greenhouse effect gasses, in as much as they use mechanical means of transportation, mainly the automobile, is undeniable. Many works agree on measuring this contribution at about 30 percent of the total greenhouse emissions. However, different means of transportation do not contribute in the same way to those emissions. Therefore, it is of the utmost importance to analyze the modal split in the travels of one population to determine the degree of participation in the environmental contamination. This paper discusses the modal split in Catalonia, based on the Everyday Mobility Survey (2006) data, from the territorial binomial urban/rural and from the motivational category that causes them.

KEY WORDS | Mobility, urban transportation, environment

Recibido el 2 de octubre de 2010, aprobado el 25 de noviembre de 2011

E-mail: carme.miralles@uab.es 


\section{Introducción}

Como ya anunció el cuarto informe del Panel Intergubernamental sobre el Cambio Climático de las Naciones Unidas en 2007, el calentamiento del sistema climático es inequívoco, como consecuencia del efecto invernadero generado por las emisiones de dióxido de carbono en la atmósfera (Intergovernmental Panel on Climate Change [IPPC], 2007). Uno de los sectores que más contribuye a estas emisiones es el transporte, por lo que parece oportuno perfilar qué circunstancias y cuáles de sus variables participan en mayor medida en el aumento de las emisiones de $\mathrm{CO}_{2}$.

La primera aproximación es que no todos los medios de transporte contribuyen del mismo modo a dichas emisiones. Algunos, como la bicicleta y el ir andando, no favorecen el incremento de los gases de efecto invernadero; en cambio, el transporte viario, especialmente el coche privado, aporta importantes cantidades de emisiones, con relación a la energía fósil que utiliza. Tales disparidades llevan a que sea fundamental analizar el reparto modal de los desplazamientos, para entender la contribución de los transportes al calentamiento global (Kaufmann, 2000; Hamilton, 2003). El objetivo de este artículo es analizar la participación que tienen los distintos modos de transporte en el total de los desplazamientos en Cataluña, una de las comunidades autónomas españolas.

$\mathrm{El}$ análisis de la estructura modal se ha realizado a partir de los datos que ofrece la Encuesta de Movilidad Cotidiana de Cataluña de 2006 (EMQ 2006). ${ }^{1}$ Esta constituye una fuente de información básica sobre los desplazamientos en la región desde la perspectiva de la población que se mueve o, lo que es lo mismo, desde la demanda y no desde la oferta de medios de transporte. Tal perspectiva permite valorar la incidencia de los transportes más contaminantes en el conjunto del reparto modal de todos los viajes, incluidos el ir andando y la bicicleta. A la vez, permite estudiar la contribución de los transportes al cambio climático desde los distintos modelos urbanos y la estructura social de la demanda de transportes.

\section{El transporte, una actividad altamente contaminante}

En la comunidad académica internacional existe un amplio consenso respecto de que los transportes contribuyen de forma notable a elevar los niveles de elementos nocivos en el medio ambiente, especialmente en el consumo de energía fósil y en las emisiones de gases de efecto invernadero, responsables del cambio climático tanto a escala local como global (Folch, 2008; Banister \& Anable, 2009; Giddens, 2009). Y aunque en los últimos años la eficiencia de los motores y la calidad del combustible han mejorado, estos avances se han diluido por el incremento del parque de vehículos y de desplazamientos, por la introducción de motores más potentes y por una menor ocupación de los vehículos (Robusté \& Casas, 2005). Paralelamente,

1 La Encuesta de Movilidad Cotidiana de Cataluña (EMQ 2006) se realizó gracias a la colaboración del Departamento de Política Territorial de la Generalitat de Cataluña y a la Autoridad del Transporte Metropolitano de Barcelona. 
empieza a existir cierta unanimidad en torno a la idea de que la participación de los transportes en la contaminación ambiental no se puede reducir solo a partir de los avances tecnológicos. Es necesario recurrir también a estrategias territoriales que afecten directamente los modelos de movilidad (Banister, 2011) y, especialmente, al reparto modal de los desplazamientos en las zonas urbanas y metropolitanas, pues no todos los medios de transporte contribuyen del mismo modo ni al consumo energético ni a las emisiones de gases contaminantes.

Los transportes son los grandes consumidores de energía fósil (Corominas, 2005), responsables de más del 35 por ciento de la demanda final de combustible en Europa (European Union, 2001). En España la situación es similar. Según datos del Ministerio de Industria, Turismo y Comercio, en 2005 el transporte consumía el 37,5 por ciento de la energía final, mientras que la industria lo hacía en 35,4 por ciento, y el resto de actividades, con 27,1 por ciento. Además, mientras la industria ha podido rebajar los niveles de consumo en los últimos veinticinco años en unos 15 puntos porcentuales, el transporte los ha aumentado en más de 10 puntos, consumo que no solo se ha incrementado en términos absolutos, sino también con relación a las personas transportadas. En Cataluña, en los primeros años de la década pasada (2001-2003), la intensidad energética del transporte (el cociente entre energía consumida y el número de viajeros transportados por kilómetro) ha pasado de un índice de 8,54 a situarse casi en 14 (Insitut d'Estadística de Catalunya [Idescat], 2003).

Sin embargo, el consumo energético no es homogéneo para todos los medios de transporte mecánicos. Al contrario, como se ve en el Gráfico 1, mientras la bicicleta tiene un consumo medio de 0,8 millones de joules por persona y kilómetro, los transportes colectivos se sitúan por encima de los 2 millones y los transportes privados superan los 4 millones.

$\mathrm{Al}$ mismo tiempo, el transporte por carretera tiene un consumo energético menos eficiente que otros medios, ya que para realizar un mismo trabajo físico (que se mide en pasajero-kilómetro) el transporte por carretera consume unos 23 gramos equivalentes de petróleo-pasajero-km, mientras que en el ferrocarril este índice baja a un nivel de 11 gramos (Agencia Ambiental Europea [AAE], 2011).

Como se ve en el Cuadro 1, en los medios de transporte existe una relación muy directa entre energía consumida y emisiones de $\mathrm{CO}_{2}$. El transporte por carretera, además de ser el que consume más toneladas equivalentes de petróleo, es el que más gases de efecto invernadero emite, alcanzando proporciones de más del 70 por ciento de las emisiones tanto en el transporte doméstico como en el total. Tales cifras derivan del uso masivo de los medios que más consumen, en el marco de una dinámica de crecimiento sostenido de los desplazamientos en los últimos años (en Cataluña, durante el primer quinquenio del siglo XXI el crecimiento ha sido del 4 por ciento anual) y de una ocupación de personas por vehículo que, en ningún caso, supera el 1,22 de media. 
GRÁFICO 1 | Consumo energético por medio de transporte en ámbito urbano (millones de joules-persona-km)

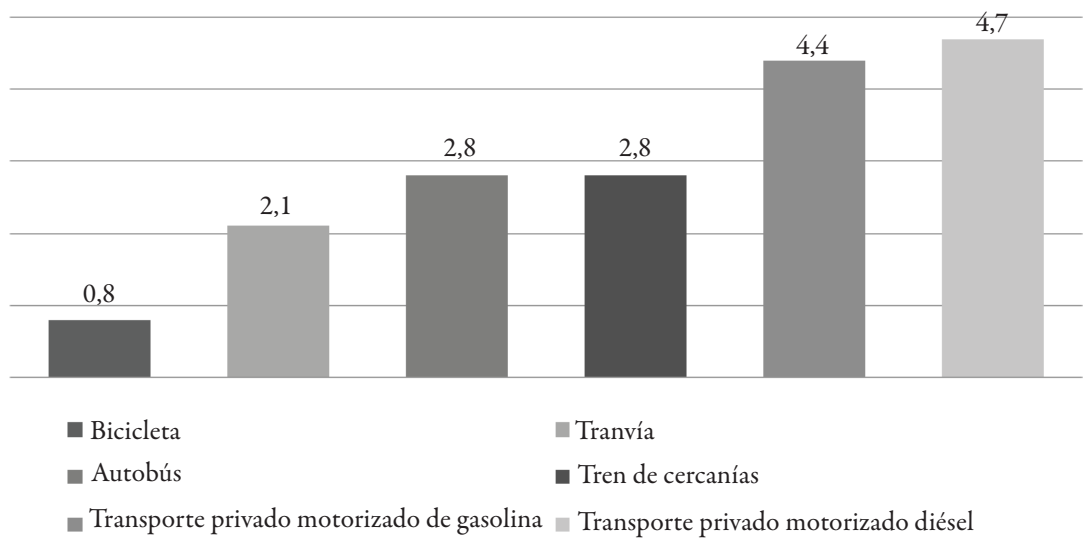

fuente International Association of Public Transport (UITP), 2009.

CUADRO 1 | Consumo de energía (millones de toneladas equivalentes de petróleo) y emisiones de $\mathrm{CO}_{2}$ (millones de toneladas) anuales de los diferentes modos de transporte en Europa (2007) (porcentajes)

\begin{tabular}{l|c|c|c}
\hline \multirow{2}{*}{ MEDIO } & \multirow{2}{*}{ ENERGía (\%) } & \multicolumn{2}{|c}{ CO $_{2}(\%)$} \\
\cline { 2 - 4 } & & DOMÉSTICO & TOTAL \\
\hline Carretera & 81,9 & 94,5 & 71,4 \\
\hline Aviación & 14,2 & 2,3 & 0,6 \\
\hline Ferrocarril & 2,5 & 0,9 & 12,6 \\
\hline Navegación & 1,4 & 2,3 & 15,4 \\
\hline
\end{tabular}

fuente Agence de l'Environnement et de la Maîtrise de l'Energie (Ademe), 2008.

Además, y como también se ha visto en el consumo, a diferencia de las emisiones procedentes del sector doméstico e industrial, las derivadas de los transportes son las que están aumentando más rápidamente. En España, entre 1990 y 2000, las emisiones de $\mathrm{CO}_{2}$ procedentes del transporte se han incrementado de 58 a 85 millones de toneladas anuales, o sea, 48 por ciento en solo una década. Buena parte de este incremento ha sido paralelo al desarrollo económico y social, a la mejora de las infraestructuras de transporte y al crecimiento de las zonas metropolitanas (Miralles-Guasch \& Cebollada, 2009). Por tanto, en el paradigma de la sostenibi- 
lidad que Europa promueve, el reto es desasociar las ratios crecientes de motorización respecto del crecimiento general de la economía (Comisión Europea, 2001 y 2007). Pues, si en la Europa de los 15, en 1990 los índices se igualaron a 100, en el año 2006 la población alcanzó 110; el PIB, 145; los viajeros por kilómetro, 160; y los gases de efecto invernadero alcanzaron los 190 (Ministerio de Medio Ambiente, 2006).

\section{Las encuestas de movilidad como fuente de información para evaluar el cambio climático en el sector de los transportes}

Para evaluar la contribución del sector transporte a los índices del gasto energético y de la contaminación atmosférica se utilizan, normalmente, datos del parque de vehículos. Sin embargo, estas fuentes de información derivadas de la oferta y centradas en un solo medio de transporte no pueden valorar la contribución del sector en su conjunto. Esto solo es posible si se la estima con relación a los modelos de movilidad del conjunto de desplazamientos de una población, por lo que parece imprescindible introducir fuentes de información desde la demanda, desde la población que se mueve, donde se contabilicen todos los desplazamientos y el conjunto de medios de transporte. En este artículo se analiza el reparto modal de la movilidad de toda la población móvil en Cataluña, incluyendo todos los motivos y medios de transporte. Este enfoque permite valorar los modos de transporte más contaminantes con relación al resto de medios, a los territorios donde se utilizan con más frecuencia y a las actividades que estos permiten realizar. A la par, subrayan el hecho de que no es la movilidad la que provoca las emisiones de $\mathrm{CO}_{2}$, sino la utilización de ciertos modos de transporte; en particular, el coche privado.

En Cataluña existe una larga tradición en el estudio de la demanda de movilidad habitual de las personas; es así que, desde 1970, esta comunidad autónoma incluyó un formulario de movilidad en el censo de población. Sin embargo, la operación estadística que ha permitido saber cómo y por qué se mueve la población, se inició recién en 2006 para el conjunto del territorio catalán. Antes de esta fecha, la información procedía de los censos de población (1981 y 2001) y de las renovaciones patronales (1986 y 1996), que ofrecían, con una periodicidad quinquenal, información sobre el primer desplazamiento del día por motivo de trabajo o estudio, lo que hoy se denomina movilidad ocupacional. $\mathrm{Y}$ aunque los datos recogidos eran muy exhaustivos, también eran muy parciales, al no incluir los desplazamientos realizados con relación a las actividades personales, como las compras cotidianas, las actividades de ocio, las visitas al médico, etcétera.

La Encuesta de Movilidad Cotidiana 2006 (EMQ 2006) partía de la experiencia de los trabajos realizados en la región metropolitana de Barcelona (RMB), ${ }^{2}$ en

2 La región metropolitana de Barcelona es el territorio metropolitano alrededor de la ciudad de Barcelona. Aunque no tiene una estructura administrativa reconocida, es utilizada para el análisis y la planificación territorial y de infraestructuras por el gobierno catalán. 
los años 1996 y 2001, cuando se elaboraron las dos primeras ediciones de la encuesta de movilidad. La novedad introducida en estos ejercicios estadísticos fue contabilizar los desplazamientos de la población desde una perspectiva más cercana a las personas que realizaban los movimientos y menos a los medios de transporte. Se trataba de conocer las características demográficas de la población móvil, cuantificar todos los motivos que generaban los desplazamientos, incluyendo los ocupacionales (relacionados con el trabajo y el estudio) y los personales (el resto de actividades), y saber en qué modos de transporte viajaba.

En la edición del año 2006 se amplió su alcance geográfico al conjunto del territorio catalán, lo que indujo algunos cambios significativos en la metodología. En primer lugar, la EMQ 2006 fue una encuesta telefónica y no presencial, como lo habían sido las anteriores. Además, la selección de unidades muestrales cambió, al sustituirse las unidades familiares por individuos. Por último, las unidades temporales de referencia semanales dieron paso a las del día anterior a la entrevista.

El objetivo de la EMQ 2006 es doble. Por una parte, caracterizar la movilidad desde los desplazamientos realizados por la población residente en el ámbito de estudio; y, por otra, analizar las relaciones territoriales que se establecen a partir de los orígenes y los destinos de los desplazamientos, teniendo en cuenta todos los flujos en el ámbito de estudio, con independencia del lugar de residencia de la población que los efectúa.

La población objeto de estudio de la EMQ 2006 es el conjunto de residentes en Cataluña de cuatro años en adelante. Incluye dos grupos, según su movilidad: profesionales de la movilidad, que engloba a todas aquellas personas que tienen la acción de desplazarse como un elemento clave en su ocupación y que declaran hacer más de siete desplazamientos diarios por motivos laborales; y población en general, que separa a la población móvil (personas que realizaron algún desplazamiento el día anterior a la entrevista) de la no móvil (personas que no salieron de casa el día preguntado). El ámbito territorial de análisis se puede desagregar a diversas escalas, todas ellas con significación estadística. Por una parte, el conjunto de Cataluña, dividida por los ámbitos territoriales funcionales definidos en el Plan Territorial de Cataluña, por comarcas y por municipios mayores de 50.000 habitantes. Además, desde el punto de vista territorial, el diseño muestral ha definido un conjunto de zonas de transporte como segmentación territorial básica en la asignación de la muestra (véase Institut d'Estudis Regionals i Metropolitans de Barcelona [IERMB], 2006).

\section{Los referentes ambientales de los transportes en Cataluña según el reparto modal}

El reparto modal de los desplazamientos de una comunidad es la referencia principal de la contribución de los transportes a la contaminación ambiental. La utilización de 
los distintos medios de transporte en los desplazamientos de la población catalana se analiza desde el binomio territorial urbano-rural en los distintos ámbitos administrativos catalanes, y desde la categoría ocupacional-personal de los motivos que los generan.

\section{Los medios de transporte utilizados en Cataluña}

De lo dicho anteriormente ha quedado claro que la utilización de los diferentes medios de transporte no es ajena a las emisiones de $\mathrm{CO}_{2}$, debido a su desigual contribución. En este sentido, es imprescindible utilizar la información que ofrece la EMQ 2006 en lo referente a los niveles de uso de los distintos medios de transporte en Cataluña, en el conjunto de desplazamientos que esta comunidad autónoma genera.

Si se analizan los tres grandes conjuntos de medios de transporte que agrupa la EMQ $2006^{3}$-el no motorizado, el privado y el público—, resulta que el más utilizado por la población catalana a lo largo de la semana es el modo no motorizado (caminar o ir en bicicleta), con un 44,5 por ciento de todos los viajes, seguido del transporte privado ( 42,5 por ciento) y del transporte público (13,0 por ciento). Estos datos, en conjunto, indican que los transportes que menos contribuyen a la contaminación atmosférica (porque, como se ha visto en el apartado anterior, generan menos emisiones) son los más utilizados por la población catalana: más del 57 por ciento de la población usa el transporte no mecánico o el público.

El análisis más desagregado de los medios de transporte utilizados muestra que el ir a pie es el modo más habitual en Cataluña donde, en un día laborable, se contabilizan unos 10,2 millones de desplazamientos de este tipo. En los modos de transporte motorizados se observa la relevancia del transporte privado, con 9,4 millones de desplazamientos al día, y un claro predominio del coche, especialmente como conductor (28,3 por ciento), lo que refleja un índice de ocupación del vehículo muy reducido (1,22 personas-coche). El transporte público concentra en un día laborable en Cataluña 3,30 millones de desplazamientos, de los cuales una cantidad similar (alrededor de un millón cada uno) se realiza en metro o en autobús. Los usuarios del ferrocarril no llegan a los 800.000 viajeros al día. Por lo tanto, y a pesar de que ir a pie es el medio de desplazamiento más utilizado, y a la vez el más sostenible, cuando se analizan los medios de transporte que consumen energía fósil y que, consecuentemente, son emisores de gases de efecto invernadero, el que tiene más presencia en un día laborable en Cataluña es el transporte privado con un solo ocupante.

\section{No en todos los territorios los transportes contaminan de igual manera}

De todos modos, los grandes números de la distribución modal amparan situaciones muy desiguales en los diferentes territorios catalanes, que derivan, básicamente, de la diversidad de la oferta de transporte público en las diferentes regiones (Figura 1). Estas

3 A lo largo de la EMQ 2006 se han considerado dieciséis medios de transporte diferentes, que se agrupan en modos no motorizados (ir a pie o en bicicleta), medios de transporte público y medios de transporte privado. 
desigualdades hacen que las características de la movilidad tengan un patrón diverso según el lugar donde se ubican, hecho que incide directamente en los desiguales referentes ambientales del transporte en Cataluña.

\section{FIGURA 1 | Regiones catalanas y municipios de más de 50.000 habitantes}

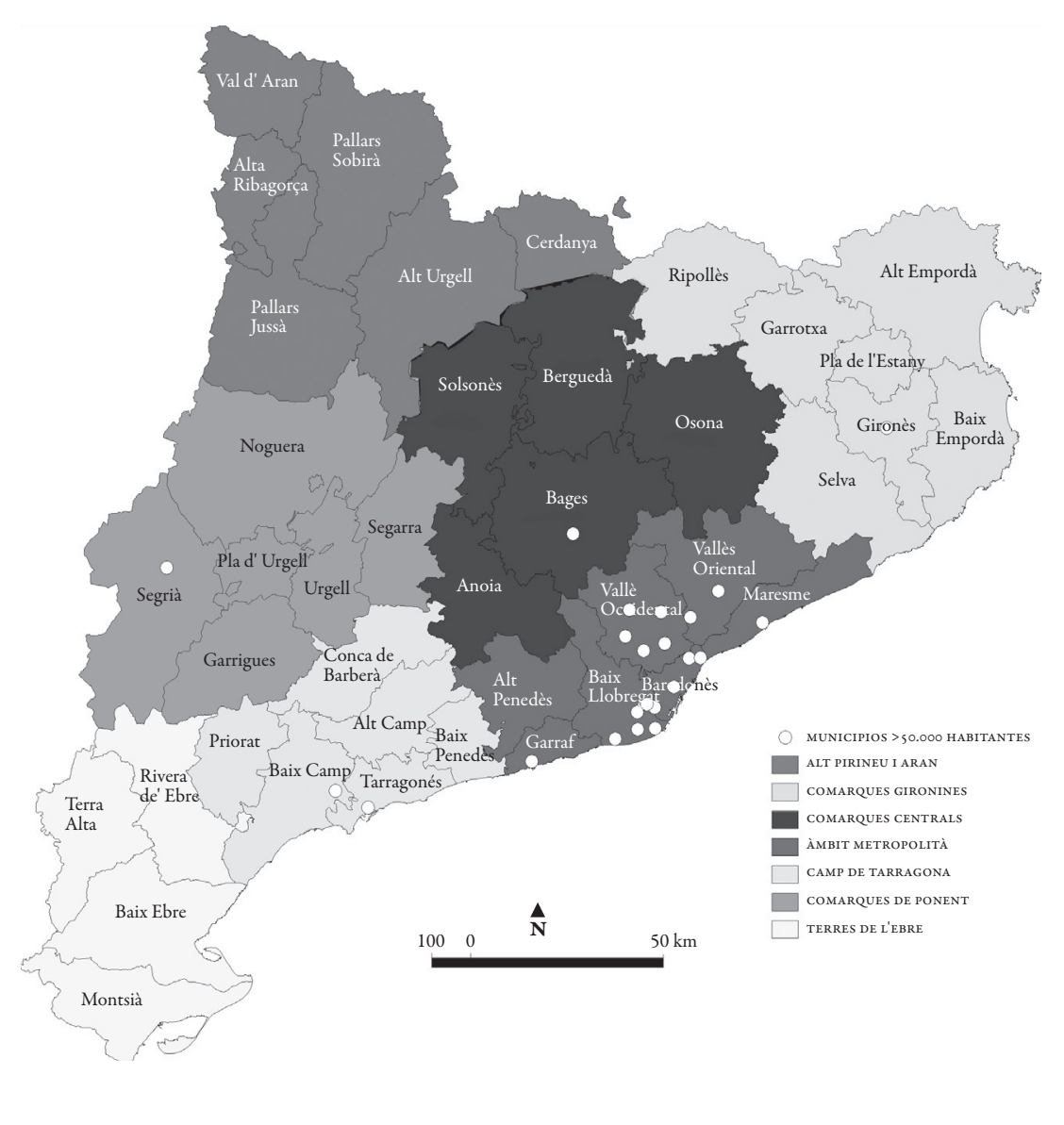

fuente Institut d'Estudis Regionals i Metropolitans de Barcelona (IERMB), 2006.

Más allá del transporte no motorizado (que oscila entre el 45,5 por ciento de la RMB y el 53,2 por ciento de las Tierras del Ebro), se pueden definir dos modelos de movilidad en Cataluña, según la proporción de uso del modo público y el privado: uno identificado con la región metropolitana de Barcelona, donde la cuota de transporte público llega casi al 20 por ciento de los desplazamientos en un día laborable y el transporte privado no supera el 36 por ciento; y otro que engloba el resto de los seis ámbitos territoriales, en los que la cuota de los viajes en 
transporte público oscila entre poco menos del 3 por ciento y el 6 por ciento del total de desplazamientos diarios, y donde el transporte privado llega casi al 50 por ciento, o incluso sobrepasa este porcentaje (Gráfico 2). Estas diferencias reflejan unos territorios más urbanos, como la RMB, y otros más rurales. En los primeros, la dimensión de los municipios, la densidad de las tramas urbanas y la tradición de uso del transporte público difieren del resto del territorio, donde la movilidad se expresa en modos individuales, sean no motorizados, como la bicicleta y el ir andando, o el coche privado.

GRÁFICo 2 Distribución de los desplazamientos en los días laborables según el medio de transporte y el ámbito de residencia

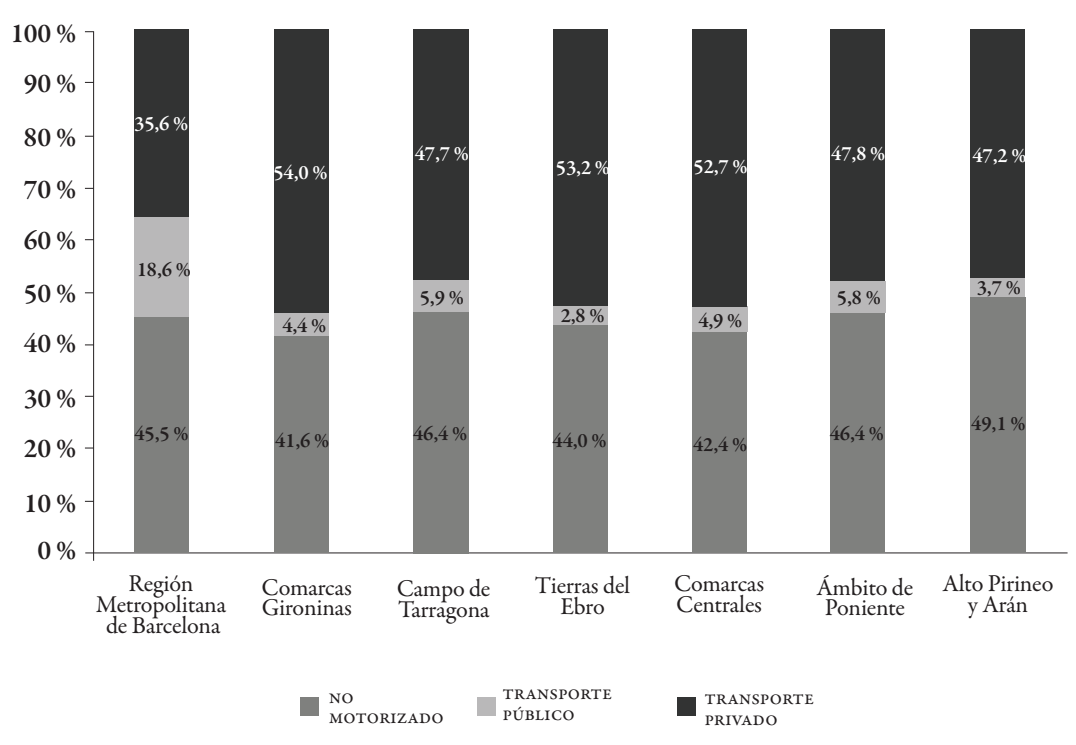

fuente Autoritat del Transport Metropolità (ATM) y Generalitat de Cataluña, 2006.

El tamaño del municipio también implica diferencias significativas. En el Cuadro 2 se observa que cuanto mayor es la ciudad, más desplazamientos no motorizados y en transporte público se hacen, y menos en transporte privado. Es significativo el caso de Barcelona, donde el 47 por ciento de los viajes en día laborable se realiza a pie o en bicicleta, alrededor del 30 por ciento en transporte público y solo el 22 por ciento en transporte privado. Esta última cifra contrasta con los municipios de menos de 10.000 habitantes, donde el 60 por ciento de los desplazamientos se hace en transporte privado. Además, el volumen de población incluido en cada una de estas tipologías de municipio es similar, por lo que el número absoluto de desplazamientos relacionados con estos porcentajes es de dimensiones comparables. 
CUADRO 2| Distribución de los desplazamientos en día laborable según el modo principal de transporte y el tamaño del municipio de residencia (porcentajes)

\begin{tabular}{c|c|c|c|c}
\hline $\begin{array}{c}\text { MENYS } \\
\text { I } 0.000 \mathrm{HAB} .\end{array}$ & $\begin{array}{c}\text { DE IO.000 A } \\
50.000 \mathrm{HAB} .\end{array}$ & $\begin{array}{c}\text { MÉS DE } \\
50.000 \mathrm{HAB} .\end{array}$ & BARCELONA & CATALUNYA \\
\hline 25,5 & 24,7 & 24,6 & 23,7 & 24,6 \\
28,6 & 29,7 & 29,9 & 31,2 & 25,1 \\
24,2 & 24,8 & 25,0 & 26,3 & 250,3 \\
\hline
\end{tabular}

fuente Autoritat del Transport Metropolità (ATM) y Generalitat de Cataluña, 2006.

\section{Los medios de transporte utilizados dependen del motivo de desplazamiento}

El medio de transporte utilizado no solo depende del lugar de residencia, sino también del motivo que genera el desplazamiento. Así, el vehículo privado es el más utilizado por motivos ocupacionales ( 51,1 por ciento), para ir a trabajar y a estudiar, mientras que los motivos personales están más asociados a los modos no motorizados (57,0 por ciento). Ello deriva, básicamente, de la opcionalidad en los destinos de las actividades más personales, como las compras o el ocio, y de la proximidad al lugar de residencia de los equipamientos escolares o asistenciales. Las ciudades compactas y mixtas como, en general, son las catalanas, ofrecen proximidad en muchos de estos desplazamientos y permiten el uso de los modos no motorizados, básicamente el caminar. En cambio, la localización del puesto de trabajo es más ajena a las voluntades de la población, lo que hace que se encuentre, normalmente, más alejado del lugar de residencia. Tal situación se ve reforzada por la localización periférica de muchos de los polígonos industriales en las distintas ciudades de Cataluña.

CUADRo 3 | Distribución de los desplazamientos según el motivo y el tamaño del municipio de residencia

\begin{tabular}{l|c|c|c|c|c}
\hline MOTIVO & $\begin{array}{c}\text { MENOS DE } \\
\text { I0.000 HAB. }\end{array}$ & $\begin{array}{c}\text { DE I0.000 A } \\
50.000 \mathrm{HAB} .\end{array}$ & $\begin{array}{c}\text { MÁS DE 50.000 } \\
\text { HAB. }\end{array}$ & BARCELONA & CATALUÑA \\
\hline $\begin{array}{l}\text { MOVILIDAD } \\
\text { OCUPACIONAL }\end{array}$ & $25,5 \%$ & $24,7 \%$ & $24,6 \%$ & $23,7 \%$ & $24,6 \%$ \\
\hline $\begin{array}{l}\text { MOVILIDAD } \\
\text { PERSONAL }\end{array}$ & $28,6 \%$ & $29,7 \%$ & $29,9 \%$ & $31,2 \%$ & $29,9 \%$ \\
\hline $\begin{array}{l}\text { VUELTA } \\
\text { PERSONAL }\end{array}$ & $24,2 \%$ & $24,8 \%$ & $25,0 \%$ & $26,3 \%$ & $25,1 \%$ \\
\hline $\begin{array}{l}\text { VUELTA } \\
\text { OCUPACIONAL }\end{array}$ & $21,7 \%$ & $20,8 \%$ & $20,5 \%$ & $18,8 \%$ & $20,4 \%$ \\
\hline \begin{tabular}{l} 
TOTAL $\%)$ \\
\hline TOTAL (N)
\end{tabular} & $100 \%$ & $100 \%$ & $100 \%$ & $100 \%$ & $100 \%$ \\
\hline
\end{tabular}

fuente Autoritat del Transport Metropolità (ATM) y Generalitat de Cataluña, 2006. 
Esta relación entre el motivo y el modo cambia según el tamaño del municipio. Como se ve en el Cuadro 3, por el motivo ocupacional y por el personal, el transporte no motorizado y el público aumentan con relación al volumen del municipio, y viceversa, con respecto del privado. También es interesante observar que la cuota de transporte público es menor en todos los casos (aunque con dimensiones diferentes) para los motivos personales, en comparación con la de ir al trabajo o a estudiar.

Todo lo anterior clarifica el lugar y el motivo por los que se utilizan los modos de transporte, respecto del consumo energético y con las emisiones de gases de efecto invernadero.

\section{Conclusiones}

El transporte es la actividad que consume la mayor parte de las energías fósiles y es la responsable de más de un tercio de las emisiones de gases de efecto invernadero a la atmósfera. Sin embargo, este sector, al tener una fuente de emisiones difusas no vinculadas a las medidas de regulación que ha supuesto el Protocolo de Kioto, en los últimos años no ha asumido ninguna estrategia de contención. Al contrario, a nivel mundial, es uno de los sectores que más ha incrementado el consumo energético y más $\mathrm{CO}_{2}$ ha emitido a la atmósfera. Tal situación se relaciona con los usos masivos de los medios de transporte más contaminantes, como son los viarios, que suponen el 81 por ciento del consumo de energía y el 93 por ciento de las emisiones del sector. Entre estos, el automóvil de uso privado es el que tiene las tasas más elevadas, al contrario del ir andando y el uso de la bicicleta, que tienen niveles de contribución a la contaminación ambiental nulos o casi nulos.

Estas diferencias entre los distintos medios de transporte significan que la participación del sector transporte al incremento del efecto invernadero depende del porcentaje de uso de los distintos medios. Es decir, es el reparto modal lo que caracteriza la movilidad de un territorio, más que la cantidad de desplazamientos que en él se dan, lo que puede influir más o menos en la contaminación atmosférica por parte del sector de los transportes.

Aquí se ha analizado este reparto modal en la comunidad autónoma de Cataluña, partiendo de las cifras de demanda de transportes que ofrece la Encuesta de Movilidad Cotidiana 2006, fuente de información muy detallada que contabiliza todos los desplazamientos que se desarrollan en el territorio catalán, por todos los motivos y medios de transporte.

Desde esta información se ha analizado el reparto modal en los siete territorios catalanes, concluyendo que, si bien existen similitudes entre ellos, en los porcentajes de desplazamientos no mecanizados (que se sitúan en torno al 50 por ciento) la utilización de los transportes públicos es muy distinta. En la región metropolitana de Barcelona - el territorio más urbano y donde existe mayor oferta de medios públicos- su cuota de utilización llega casi al 20 por ciento. Si a este porcentaje se 
suma el correspondiente al transporte no mecanizado, se llega casi al 65 por ciento de los desplazamientos, lo que permite afirmar que este ámbito tiene niveles bajos de emisiones de gases de efecto invernadero, con relación a la cantidad de desplazamientos que produce.

También las razones que generan los desplazamientos tienen repartos modales distintos. Es en los desplazamientos ocasionados por motivos relacionados con el trabajo donde se utiliza más el vehículo privado, lo que significa que la distribución periférica y suburbana de los polígonos industriales en nuestras urbes, sin medios de transporte colectivo característicos de las ciudades catalanas (Miralles-Guasch \& Donat, 2007), contribuye sustancialmente a los índices de contaminación ambiental. @EURE

\section{Referencias bibliográficas}

Agence de l'Environnement et de la Maîtrise de l'Energie (Ademe). (2008). Efficacité énergétique et environnementale des modes de transport. Coordinado por Eric Vidalenc. Valbonne: Autor. Agencia Ambiental Europea (2003-2007). http://www.eea.europa.eu

Alegre, Ll. (2008). L'economia de la mobilitat. Els costos reals del transport. En P. Noy (Dir.), Mobilitats 2008. Realitats, tendències i reptes de la mobilitat a Catalunya (pp. 74-83). Barcelona: Fundació Mobilitat Sostenible i Segura.

Autoritat de Transport Metropolità (ATM) \& Generalitat de Catalunya (2006). Enquesta Mobilitat Quotidiana de Catalunya 2006. Barcelona: Autor.

Banister, D. (2011). Cities, mobility and climate change. Journal of Transport Geograhy, 19(6), 15381546.

Banister, D. \& Anable, J. (2009). Transport policies and climate chang. En S. Davouidi \& J. Crawford (Eds.), Planning for climate change: Strategies for mitigation and adaptation for spatial planners (pp. 55-69). Londres: Earthscan.

Colegio de Ingenieros de Caminos, Canales y Puertos / Comisión de Transportes (2010). Libro verde del transporte y cambio climático. Madrid: Autor.

Comisión Europea (2001). Libro blanco. La política europea del transporte de cara al 2010: la hora de la verdad. Bruselas: Comisión de las Comunidades Europeas.

Comisión Europea (2007). Libro verde. Hacia una nueva cultura de la movilidad urbana. Bruselas: Comisión de las Comunidades Europea.

Corominas, J. (2005). Energia. En J. E. Llebot (Ed.), Informe del canvi climàtic a Catalunya (pp. 229-265). Barcelona: Generalitat de Catalunya, Consell Assessor per al desenvolupament sostenible.

Directrius Nacionals de Mobilitat (DNM). (2006.) Generalitat de Catalunya. Departament de Politica Territorial i Obres Públiques (PTOP). Barcelona: PTOP. En http://bit.ly/MHY7CY

European Union (2001). White paper. European transport policy for 2010: Time to decide. Luxemburgo: European Commission.

Folch, R. (2008). Energia i sostenibilitat. Catalunya 2030. En A. Àvila \& J. Terradas (Eds.), Aula d'Ecologia. Cicle de conferències 2007 (pp. 49-56). Bellaterra: Universitat Autónoma de Barcelona, Servei de Publicacions. 
Generalitat de Catalunya, Departament de Política Territorial i Obres Públiques (PTOP). (2001). Els costos social i ambientals del transport de passatgers a Catalunya. Barcelona: Autor.

Giddens, A. (2009). The politics of climate change. Cambridge: Polity Press Ltd. Versión en español: La politica del cambio climático. Madrid: Alianza Editorial, 2010.

Hamilton C. (2003). Climate change. En D. A. Hensher \& K. J. Button (Eds.), Handbook of Transport \& the Environment (pp. 37-60). Vol. 4 de Handbooks in Transport. Amsterdam: Elsevier.

Insitut d'Estadística de Catalunya (Idescat). (2003). http:// www.idescat.cat/

Institut d'Estudis Regionals i Metropolitans de Barcelona (IERMB). (2006). Encuesta de Movilidad Cotidiana 2006. En http://www.iermb.uab.es/htm/mobilitat/esp/emq-06.asp

Intergovernmental Panel on Climate Change (IPCC). (2008). Climate change 2007 - Impacts, adaptation and vulnerability [Working Group II contribution to the Fourth Assessment Report of the IPCC]. Cambridge: Cambridge University Press.

International Association of Public Transport (UITP). http://www.uitp.org/

Kaufmann, V. (2000). Mobilité quotidienne et dynamiques urbaines. La question du rapport modal. Lausana: Presses Polytechniques et Universitaires Romandes.

Llebot, E. (Ed.) (2010). Segundo informe del cambio climático en Catalunya. Barcelona: Generalitat de Catalunya.

Ministerio de Medio Ambiente (2006). http://www.ministeriodemedioambiente.es (actualmente, Ministerio de Agricultura, Alimentación y Medio Ambiente, http://www.magrama.gob. es/es/)

Miralles-Guasch, C. \& Cebollada, A. (2003). Movilidad y transporte. Opciones politicas para la ciudad. Madrid. Fundación Altenativas.

Miralles-Guasch, C. \& Cebollada, A. (2009). Movilidad cotidiana y sostenibilidad. Una interpretación desde la geografía humana. Boletin de Asociación de Geógrafos Españoles (AGE), 50, 193-216. En http://age.ieg.csic.es/boletin/50/08 por ciento20MIRALLES.pdf

Miralles-Guasch, C. \& Donat, C. (2007). Anàlisi de l'oferta i la demanda de polígonsd'activitat a Catalunya. Papers. Regió Metropolitana de Barcelona, 45, 8-36.

Noy, P. (2008). L'eficiència energètica en la mobilitat quotidiana. En P. Noy (Dir.), Mobilitats 2008. Realitats, tendències i reptes de la mobilitat a Catalunya (pp. 48-57). Barcelona: Fundació Mobilitat Sostenible i Segura.

Pérez Martínez, P. J.; Monzón de Cáceres, A. (2008). Consumo de energía por el transporte en España y tendencias de emisión. Observatorio Medioambiental, 11, 127-147.

Robusté, F. \& Casas Esplugas, C. (2005). Transport. En J. E. Llebot, Informe del canvi climátic a Catalunya (pp. 307-337). Barcelona: Generalitat de Catalunya, Consell Assessor per al Desenvolupament Sostenible de Catalunya.

Vivier, J. (2001). The millennium cities database for sustainable transport [CD para computador]. Bruselas: Union International des Transports Publics (UITP). 